CUBO A Mathematical Journal

Vol.20, $N^{\underline{O}}$ 01, (01-16). March 2018

\title{
Approximation by Shift Invariant Univariate Sublinear-Shilkret Operators
}

\author{
George A. Anastassiou \\ Department of Mathematical Sciences, \\ University of Memphis, \\ Memphis, TN 38152, U.S.A. \\ ganastss@memphis.edu
}

\begin{abstract}
A very general positive sublinear Shilkret integral type operator is given through a convolution-like iteration of another general positive sublinear operator with a scaling type function. For it sufficient conditions are given for shift invariance, preservation of global smoothness, convergence to the unit with rates. Additionally, two examples of very general specialized operators are presented fulfilling all the above properties, the higher order of approximation of these operators is also considered.
\end{abstract}

\section{RESUMEN}

Un operador muy general positivo sublineal de tipo integral de Shilkret es dado a través de un iteración de tipo convolución de otro operador general positivo sublineal con una función de tipo escalamiento. Para estos operadores, se entregan condiciones suficientes para invariancia por shifts, conservación de la suavidad global y convergencia a la unidad con tasas. Adicionalmente, se presentan dos ejemplos de operadores muy generales especializados que satisfacen todas las propiedades anteriores, también considerando el alto orden de aproximación de estos operadores.

Keywords and Phrases: Jackson type inequality, Shilkret integral, modulus of continuity, shift invariant, global smoothness preservation, quantitative approximation.

2010 AMS Mathematics Subject Classification: 41A17, 41A25, 41A35, 41A36. 


\section{Introduction}

Let $X, Y$ be function spaces of functions from $\mathbb{R}$ into $\mathbb{R}_{+}$. Let $L_{N}: X \rightarrow Y, N \in \mathbb{N}$, be a sequence of operators with the following properties:

(i) (positive homogeneous)

$$
\mathrm{L}_{\mathrm{N}}(\alpha \mathrm{f})=\alpha \mathrm{L}_{\mathrm{N}}(\mathrm{f}), \forall \alpha \geq 0, \forall \mathrm{f} \in \mathrm{X} .
$$

(ii) (Monotonicity) if $f, g \in X$ satisfy $f \leq g$, then $L_{N}(f) \leq L_{N}(g), \forall N \in \mathbb{N}$, and

(iii) (Subadditivity)

$$
\mathrm{L}_{N}(f+g) \leq \mathrm{L}_{N}(f)+\mathrm{L}_{N}(g), \quad \forall f, g \in X
$$

We call $\mathrm{L}_{\mathrm{N}}$ positive sublinear operators.

In this article we deal with sequences of Shilkret positive sublinear operators that are constructed, with the help of Shilkret integral ([5]). Our functions spaces are continuous functions from $\mathbb{R}$ into $\mathbb{R}_{+}$. The sequence of operators is generated by a basic operator via dilated translations of convolution type using the Shilkret integral. We prove that our operators possess the following properties: of shift invariance of global smoothness preservation, of convergence to the unit operator with rates. Then we apply our results to two specific families of such Shilkret type operators.

We continue with the higher order of approximation study of these specific operators, and all results are quantitative.

Earlier similar studies have been done by the author, see [3], Chapters 10-17, and 2, Chapters 16, 17. These serve as motivation and inspiration to this work.

\section{Background}

Here we follow [5].

Let $\mathcal{F}$ be a $\sigma$-field of subsets of an arbitrary set $\Omega$. An extended non-negative real valued function $\mu$ on $\mathcal{F}$ is called maxitive if $\mu(\varnothing)=0$ and

$$
\mu\left(\cup_{i \in I} E_{i}\right)=\sup _{i \in I} \mu\left(E_{i}\right),
$$

where the set $I$ is of cardinality at most countable, where $\left\{E_{i}\right\}_{i \in I}$ is a disjoint collection of sets from $\mathcal{F}$. We notice that $\mu$ is monotone and $(1)$ is true even $\left\{E_{i}\right\}_{i \in I}$ are not disjoint. For more properties of $\mu$ see [5]. We also call $\mu$ a maxitive measure. Here $f$ stands for a non-negative measurable 
function on $\Omega$. In [5], Niel Shilkret developed his non-additive integral defined as follows:

$$
\left(N^{*}\right) \int_{D} f d \mu:=\sup _{y \in Y}\{y \cdot \mu(D \cap\{f \geq y\})\}
$$

where $Y=[0, m]$ or $Y=[0, m)$ with $0<m \leq \infty$, and $D \in \mathcal{F}$. Here we take $Y=[0, \infty)$.

It is easily proved that

$$
\left(N^{*}\right) \int_{D} f d \mu=\sup _{y>0}\{y \cdot \mu(D \cap\{f>y\})\} .
$$

The Shilkret integral takes values in $[0, \infty]$.

The Shilkret integral ([5]) has the following properties:

$$
\left(N^{*}\right) \int_{\Omega} \chi_{E} d \mu=\mu(E)
$$

where $\chi_{E}$ is the indicator function on $E \in \mathcal{F}$,

$$
\begin{aligned}
& \left(N^{*}\right) \int_{D} \operatorname{cfd} \mu=c\left(N^{*}\right) \int_{D} f d \mu, \quad c \geq 0, \\
& \left(N^{*}\right) \int_{D} \sup _{n \in \mathbb{N}} d \mu=\sup _{n \in \mathbb{N}}\left(N^{*}\right) \int_{D} f_{n} d \mu,
\end{aligned}
$$

where $f_{n}, n \in \mathbb{N}$, is an increasing sequence of elementary (countably valued) functions converging uniformly to $f$. Furthermore we have

$$
\begin{gathered}
\left(N^{*}\right) \int_{D} f d \mu \geq 0, \\
f \geq g \text { implies }\left(N^{*}\right) \int_{D} f d \mu \geq\left(N^{*}\right) \int_{D} g d \mu,
\end{gathered}
$$

where $f, g: \Omega \rightarrow[0, \infty]$ are measurable.

Let $a \leq f(\omega) \leq b$ for almost every $\omega \in E$, then

$$
\begin{gathered}
a \mu(E) \leq\left(N^{*}\right) \int_{E} f d \mu \leq b \mu(E) ; \\
\left(N^{*}\right) \int_{E} 1 d \mu=\mu(E)
\end{gathered}
$$

$f>0$ almost everywhere and $\left(N^{*}\right) \int_{E} f d \mu=0$ imply $\mu(E)=0$;

$\left(N^{*}\right) \int_{\Omega} f d \mu=0$ if and only $f=0$ almost everywhere;

$\left(N^{*}\right) \int_{\Omega} f d \mu<\infty$ implies that

$$
\bar{N}(f):=\{\omega \in \Omega \mid f(\omega) \neq 0\} \text { has } \sigma \text {-finite measure; }
$$




$$
\left(N^{*}\right) \int_{D}(f+g) d \mu \leq\left(N^{*}\right) \int_{D} f d \mu+\left(N^{*}\right) \int_{D} g d \mu
$$

and

$$
\left|\left(N^{*}\right) \int_{D} f d \mu-\left(N^{*}\right) \int_{D} g d \mu\right| \leq\left(N^{*}\right) \int_{D}|f-g| d \mu .
$$

From now on in this article we assume that $\mu: \mathcal{F} \rightarrow[0,+\infty)$.

\section{Univariate Theory}

This section is motivated and inspired by [3] and [4].

Let $\mathcal{L}$ be the Lebesgue $\sigma$ - algebra on $\mathbb{R}$, and the set function $\mu: \mathcal{L} \rightarrow[0,+\infty]$, which is assumed to be maxitive. Let $\mathrm{C}_{\mathrm{U}}\left(\mathbb{R}, \mathbb{R}_{+}\right)$be the space of uniformly continuous functions from $\mathbb{R}$ into $\mathbb{R}_{+}$, and $\mathrm{C}\left(\mathbb{R}, \mathbb{R}_{+}\right)$the space of continuous functions from $\mathbb{R}$ into $\mathbb{R}_{+}$. For any $f \in \mathrm{C}_{\mathrm{u}}\left(\mathbb{R}_{,}, \mathbb{R}_{+}\right)$ we have $\omega_{1}(f, \delta)<+\infty, \delta>0$, where

$$
\omega_{1}(f, \delta):=\sup _{\substack{x, y \in \mathbb{R}: \\|x-y| \leq \delta}}|f(x)-f(y)|, \delta>0
$$

is the first modulus of continuity.

Let $\left\{t_{k}\right\}_{k \in \mathbb{Z}}$ be a sequence of positive sublinear operators that map $C_{u}\left(\mathbb{R}, \mathbb{R}_{+}\right)$into $C\left(\mathbb{R}, \mathbb{R}_{+}\right)$ with the property

$$
\left(\mathrm{t}_{\mathrm{k}}(\mathrm{f})\right)(\mathrm{x}):=\mathrm{l}_{\mathrm{o}}\left(\mathrm{f}\left(2^{-\mathrm{k}} \cdot\right)\right)(\mathrm{x}), \quad \forall \mathrm{x} \in \mathbb{R}, \forall \mathrm{f} \in \mathrm{C}_{\mathrm{u}}\left(\mathbb{R}, \mathbb{R}_{+}\right) .
$$

For a fixed $a>0$ we assume that

$$
\sup _{\substack{u, y \in \mathbb{R}: \\|\mathfrak{u}-\mathbf{y}| \leq \mathfrak{a}}}\left|t_{0}(f, u)-f(y)\right| \leq \omega_{1}\left(f, \frac{m a+n}{2^{r}}\right), \forall f \in C_{u}\left(\mathbb{R}, \mathbb{R}_{+}\right),
$$

where $\mathrm{m} \in \mathbb{N}, n \in \mathbb{Z}_{+}, r \in \mathbb{Z}$.

Let $\psi: \mathbb{R} \rightarrow \mathbb{R}_{+}$which is Lebesgue measurable, such that

$$
\left(N^{*}\right) \int_{-a}^{a} \psi(u) d \mu(u)=1 .
$$

We define the positive sublinear-Shilkret operators

$$
\left(T_{0}(f)\right)(x):=\left(N^{*}\right) \int_{-a}^{a}\left(t_{0} f\right)(x-u) \psi(u) d \mu(u),
$$

and

$$
\left(T_{k}(f)\right)(x):=\left(T_{0}\left(f\left(2^{-k} \cdot\right)\right)\right)\left(2^{k} x\right), \quad \forall k \in \mathbb{Z}, \forall x \in \mathbb{R} .
$$


Therefore it holds

$$
\begin{gathered}
\left(T_{k}(f)\right)(x)=\left(N^{*}\right) \int_{-a}^{a}\left(t_{0}\left(f\left(2^{-k} \cdot\right)\right)\right)\left(2^{k} x-u\right) \psi(u) d \mu(u)= \\
\left(N^{*}\right) \int_{-a}^{a}\left(t_{k}(f)\right)\left(2^{k} x-u\right) \psi(u) d \mu(u)
\end{gathered}
$$

$\forall x \in \mathbb{R}, \forall k \in \mathbb{Z}$

Indeed here we have

$$
\begin{gathered}
\left(T_{k}(f)\right)(x) \stackrel{\sqrt[8]{8}}{\leq}\left(N^{*}\right) \int_{-a}^{a}\left\|t_{k}(f)\left(2^{k} x-\cdot\right)\right\|_{\infty,[-a, a]} \psi(u) d \mu(u) \stackrel{(55)}{=} \\
\left\|t_{k}(f)\left(2^{k} x-\cdot\right)\right\|_{\infty,[-a, a]}\left(\left(N^{*}\right) \int_{-a}^{a} \psi(u) d \mu(u)\right)= \\
\left\|t_{k}(f)\left(2^{k} x-\cdot\right)\right\|_{\infty,[-a, a]}<+\infty .
\end{gathered}
$$

Hence $\left(T_{k}(f)\right)(x) \in \mathbb{R}_{+}$is well-defined.

Let $\mathrm{f}, \mathrm{g} \in \mathcal{M}\left(\mathbb{R}, \mathbb{R}_{+}\right)$(Lebesgue measurable functions) where $X \in A, A \subset \mathbb{R}$ is a Lebesgue measurable set.

We derive that

$$
\left|\left(N^{*}\right) \int_{A} f(x) d \mu(x)-N^{*} \int_{A} g(x) d \mu(x)\right| \stackrel{\sqrt[12]{\leq}}{\leq}\left(N^{*}\right) \int_{A}|f(x)-g(x)| d \mu(x) .
$$

We need

Definition 3.1. Let $\mathrm{f}_{\alpha}(\cdot):=\mathrm{f}(\cdot+\alpha), \alpha \in \mathbb{R}$, and $\Phi$ be an operator. If $\Phi\left(\mathrm{f}_{\alpha}\right)=(\Phi \mathrm{f})_{\alpha}$, then $\Phi$ is called a shift invariant operator.

We give

Theorem 3.2. Assume that

$$
\left(t_{0}\left(f\left(2^{-k} \cdot+\alpha\right)\right)\right)\left(2^{k} u\right)=\left(t_{0}\left(f\left(2^{-k} \cdot\right)\right)\right)\left(2^{k}(u+\alpha)\right),
$$

for all $\mathrm{k} \in \mathbb{Z}, \alpha \in \mathbb{R}$ fixed, all $\mathrm{u} \in \mathbb{R}$ and any $\mathrm{f} \in \mathrm{C}_{\mathrm{u}}\left(\mathbb{R}, \mathbb{R}_{+}\right)$. Then $\mathrm{T}_{\mathrm{k}}$ is a shift invariant operator for all $k \in \mathbb{Z}$.

Proof. We have that

$$
\begin{gathered}
\left(T_{k}(f(\cdot+\alpha))\right)(x)=\left(T_{k}\left(f_{\alpha}\right)\right)(x) \stackrel{\sqrt[18]{=}}{=} \\
\left(N^{*}\right) \int_{-a}^{a}\left(t_{0}\left(f_{\alpha}\left(2^{-k} \cdot\right)\right)\right)\left(2^{k} x-u\right) \psi(u) d \mu(u)= \\
\left(N^{*}\right) \int_{-a}^{a}\left(t_{0}\left(f\left(2^{-k} \cdot+\alpha\right)\right)\right)\left(2^{k} x-u\right) \psi(u) d \mu(u)=
\end{gathered}
$$




$$
\begin{gathered}
\left(N^{*}\right) \int_{-a}^{a}\left(t_{0}\left(f\left(2^{-k} \cdot+\alpha\right)\right)\right)\left(2^{k}\left(x-2^{-k} u\right)\right) \psi(u) d \mu(u) \stackrel{\sqrt{21}=}{=} \\
\left(N^{*}\right) \int_{-a}^{a}\left(t_{0}\left(f\left(2^{-k} \cdot\right)\right)\right)\left(2^{k}\left(x-2^{-k} u+\alpha\right)\right) \psi(u) d \mu(u)= \\
\left(N^{*}\right) \int_{-a}^{a}\left(t_{0}\left(f\left(2^{-k} \cdot\right)\right)\right)\left(2^{k}(x+\alpha)-u\right) \psi(u) d \mu(u) \stackrel{(18)}{=}\left(T_{k}(f)\right)(x+\alpha),
\end{gathered}
$$

that is

$$
T_{k}\left(f_{\alpha}\right)=\left(T_{k}(f)\right)_{\alpha}
$$

proving the claim.

It follows the global smoothness of the operators $T_{k}$.

Theorem 3.3. For any $\mathrm{f} \in \mathrm{C}_{\mathrm{u}}\left(\mathbb{R}, \mathbb{R}_{+}\right)$assume that, for all $\mathrm{u} \in \mathbb{R}$,

$$
\left|\left(t_{0}(f)\right)(x-u)-\left(t_{0}(f)\right)(y-u)\right| \leq \omega_{1}(f,|x-y|),
$$

for any $\mathrm{x}, \mathrm{y} \in \mathbb{R}$. Then

$$
\omega_{1}\left(T_{k} f, \delta\right) \leq \omega_{1}(f, \delta), \quad \forall \delta>0
$$

Proof. We observe that

$$
\begin{gathered}
\left|\left(T_{0}(f)\right)(x)-\left(T_{0}(f)\right)(y)\right|= \\
\left|\left(N^{*}\right) \int_{-a}^{a}\left(t_{0} f\right)(x-u) \psi(u) d \mu(u)-\left(N^{*}\right) \int_{-a}^{a}\left(t_{0} f\right)(y-u) \psi(u) d \mu(u)\right| \stackrel{(20)}{\leq} \\
\left(N^{*}\right) \int_{-a}^{a}\left|\left(t_{0} f\right)(x-u)-\left(t_{0} f\right)(y-u)\right| \psi(u) d \mu(u) \stackrel{(b y)(24),(50))}{\leq} \\
\omega_{1}(f,|x-y|)\left(\left(N^{*}\right) \int_{-a}^{a} \psi(u) d \mu(u)\right) \stackrel{(15)}{=} \omega_{1}(f,|x-y|) .
\end{gathered}
$$

So that

$$
\left|\left(T_{0}(f)\right)(x)-\left(T_{0}(f)\right)(y)\right| \leq w_{1}(f,|x-y|) .
$$

From (17), (27) we get

$$
\begin{gathered}
\left|\left(T_{k}(f)\right)(x)-\left(T_{k}(f)\right)(y)\right| \stackrel{\sqrt{17}}{=} \\
\left|\left(T_{0}\left(f\left(2^{-k} \cdot\right)\right)\right)\left(2^{k} x\right)-\left(T_{0}\left(f\left(2^{-k} \cdot\right)\right)\right)\left(2^{k} y\right)\right| \leq \\
\omega_{1}\left(f\left(2^{-k} \cdot\right), 2^{k}|x-y|\right)=\omega_{1}(f,|x-y|),
\end{gathered}
$$

i.e. global smoothness for $T_{k}$ has been proved.

The convergence of $T_{k}$ to the unit operator, as $k \rightarrow+\infty, k$ with rates follows: 
Theorem 3.4. For $\mathrm{f} \in \mathrm{C}_{\mathrm{u}}\left(\mathbb{R}, \mathbb{R}_{+}\right)$, under the assumption (14), we have

$$
\left|\left(T_{k}(f)\right)(x)-f(x)\right| \leq \omega_{1}\left(f, \frac{m a+n}{2^{k+r}}\right),
$$

where $\mathrm{m} \in \mathbb{N}, \mathrm{n} \in \mathbb{Z}_{+}, \mathrm{k}, \mathrm{r} \in \mathbb{Z}$.

Proof. We notice that

$$
\begin{aligned}
& \left|\left(T_{k}(f)\right)(x)-f(x)\right| \stackrel{(17)}{=}\left|\left(T_{0}\left(f\left(2^{-k} \cdot\right)\right)\right)\left(2^{k} x\right)-f(x)\right| \stackrel{(18)}{=} \\
& \left|\left(N^{*}\right) \int_{-a}^{a}\left(t_{0}\left(f\left(2^{-k} \cdot\right)\right)\right)\left(2^{k} x-u\right) \psi(u) d \mu(u)-f(x)\right| \stackrel{(15 x)}{=} \\
& \left|\left(N^{*}\right) \int_{-a}^{a}\left(t_{0}\left(f\left(2^{-k} \cdot\right)\right)\right)\left(2^{k} x-u\right) \psi(u) d \mu(u)-\left(N^{*}\right) \int_{-a}^{a} f(x) \psi(u) d \mu(u)\right| \stackrel{\sqrt[20]{\leq}}{\leq} \\
& \left(N^{*}\right) \int_{-a}^{a}\left|\left(t_{0}\left(f\left(2^{-k} \cdot\right)\right)\right)\left(2^{k} x-u\right)-f(x)\right| \psi(u) d \mu(u)= \\
& \left(N^{*}\right) \int_{-a}^{a}\left|\left(t_{0}\left(f\left(2^{-k} \cdot\right)\right)\right)\left(2^{k} x-u\right)-f\left(2^{-k} \cdot\right)\left(2^{k} x\right)\right| \psi(u) d \mu(u) \stackrel{\sqrt[14]{\leq}}{\leq} \\
& \text { (here } \left.\left|\left(2^{k} x-u\right)-2^{k} x\right|=|u| \leq a\right) \\
& \omega_{1}\left(f\left(2^{-k} \cdot\right), \frac{m a+n}{2^{r}}\right)\left(\left(N^{*}\right) \int_{-a}^{a} \psi(u) d \mu(u)\right) \stackrel{15}{=} \\
& \omega_{1}\left(f\left(2^{-k} \cdot\right), \frac{m a+n}{2^{r}}\right) \cdot 1=\omega_{1}\left(f, \frac{m a+n}{2^{k+r}}\right) \text {, }
\end{aligned}
$$

proving the claim.

We give some applications.

For each $k \in \mathbb{Z}$, we define

(i)

$$
\left(B_{k} f\right)(x):=\left(N^{*}\right) \int_{-a}^{a} f\left(x-\frac{u}{2^{k}}\right) \psi(u) d \mu(u)
$$

i.e., here

$$
\begin{gathered}
\left(t_{k}(f)\right)(u)=f\left(\frac{u}{2^{k}}\right), \\
\text { and } \\
\left(t_{0}(f)\right)(u)=f(u),
\end{gathered}
$$

are continuous in $\mathfrak{u} \in \mathbb{R}$.

Also for $k \in \mathbb{Z}$, we define

(ii)

$$
\left(\Gamma_{k}(f)\right)(x):=\left(N^{*}\right) \int_{-a}^{a} \gamma_{k}^{f}\left(2^{k} x-u\right) \psi(u) d \mu(u),
$$


where

$$
\left(t_{k}(f)\right)(u)=\gamma_{k}^{f}(u):=\sum_{j=0}^{n} w_{j} f\left(\frac{u}{2^{k}}+\frac{j}{2^{k} n}\right)
$$

$\mathrm{n} \in \mathbb{N}, w_{j} \geq 0, \sum_{j=0}^{n} w_{j}=1$,

is continuous in $u \in \mathbb{R}$.

Notice here that

$$
\left(t_{0}(f)\right)(u)=\gamma_{0}^{f}(u)=\sum_{j=0}^{n} w_{j} f\left(u+\frac{j}{n}\right)
$$

is also continuous in $u \in \mathbb{R}$.

Indeed we have

$$
\left(\Gamma_{k}(f)\right)(x)=\left(N^{*}\right) \int_{-a}^{a}\left[\sum_{j=0}^{n} w_{j} f\left(\left(x-\frac{u}{2^{k}}\right)+\frac{j}{2^{k} n}\right)\right] \psi(u) d \mu(u) .
$$

Clealry here we have

$$
\begin{aligned}
&\left(B_{k}(f)\right)(x)=\left(B_{0}\left(f\left(2^{-k} \cdot\right)\right)\right)\left(2^{k} x\right), \\
& \quad \text { and } \\
&\left(\Gamma_{k}(f)\right)(x)=\left(\Gamma_{0}\left(f\left(2^{-k} \cdot\right)\right)\right)\left(2^{k} x\right),
\end{aligned}
$$

$\forall \mathrm{k} \in \mathbb{Z}, \forall x \in \mathbb{R}$.

We give

Proposition 3.5. $\mathrm{B}_{\mathrm{k}}, \Gamma_{\mathrm{k}}$ are shift invariant operators.

Proof. (i) For $B_{k}$ operators: Here $t_{0} f=f$. Hence

$$
\begin{gathered}
\left(t_{0}\left(f\left(2^{-k} \cdot+\alpha\right)\right)\right)\left(2^{k} u\right)=f\left(2^{-k} 2^{k} u+\alpha\right)=f(u+\alpha)= \\
\left(t_{0}\left(f\left(2^{-k} \cdot\right)\right)\right)\left(2^{k}(u+\alpha)\right) .
\end{gathered}
$$

(ii) For $\Gamma_{k}$ operators:

$$
\left(t_{0}(f)\right)(u)=\sum_{j=0}^{n} w_{j} f\left(u+\frac{j}{n}\right)
$$

Hence

$$
\begin{aligned}
& \left(t_{0}\left(f\left(2^{-k} \cdot+\alpha\right)\right)\right)\left(2^{k} u\right)=\sum_{j=0}^{n} w_{j} f\left(2^{-k}\left(2^{k} u+\frac{j}{n}\right)+\alpha\right)= \\
& \sum_{j=0}^{n} w_{j} f\left(2^{-k}\left(2^{k}(u+\alpha)+\frac{j}{n}\right)\right)=\left(t_{0}\left(f\left(2^{-k} \cdot\right)\right)\right)\left(2^{k}(u+\alpha)\right),
\end{aligned}
$$

proving the claim. 
Next we show that the operators $B_{k}, \Gamma_{k}$ possess the property of global smoothness preservation.

Theorem 3.6. For all $\mathrm{f} \in \mathrm{C}_{\mathrm{u}}\left(\mathbb{R}, \mathbb{R}_{+}\right)$and all $\delta>0$ we have

$$
\begin{aligned}
& \omega_{1}\left(B_{k} f, \delta\right) \leq \omega_{1}(f, \delta), \\
& \text { and } \\
& \omega_{1}\left(\Gamma_{k} f, \delta\right) \leq \omega_{1}(f, \delta) .
\end{aligned}
$$

Proof. (i) For $B_{k}$ operators: Here $t_{0} f=f$, therefore

$$
\left|\left(t_{0}(f)\right)(x-u)-\left(t_{0}(f)\right)(y-u)\right|=|f(x-u)-f(y-u)| \leq w_{1}(f,|x-y|) .
$$

(ii) For $\Gamma_{k}$ operators: We observe that

$$
\begin{gathered}
\left|\left(t_{0}(f)\right)(x-u)-\left(t_{0}(f)\right)(y-u)\right|=\left|\gamma_{0}^{f}(x-u)-\gamma_{0}^{f}(y-u)\right|= \\
\left|\sum_{j=0}^{n} w_{j}\left(f\left(x-u+\frac{j}{n}\right)-f\left(y-u+\frac{j}{n}\right)\right)\right| \leq \\
\quad \sum_{j=0}^{n} w_{j}\left|f\left(x-u+\frac{j}{n}\right)-f\left(y-u+\frac{j}{n}\right)\right| \leq \\
\omega_{1}(f,|x-y|)\left(\sum_{j=0}^{n} w_{j}\right)=w_{1}(f,|x-y|),
\end{gathered}
$$

proving the claim.

The operators $B_{k}, \Gamma_{k}, k \in \mathbb{Z}$, converge to the unit operator with rates presented next.

Theorem 3.7. For $\mathrm{k} \in \mathbb{Z}$,

$$
\begin{gathered}
\left|\left(B_{k}(f)\right)(x)-f(x)\right| \leq \omega_{1}\left(f, \frac{a}{2^{k}}\right), \\
\quad \text { and } \\
\left|\left(\Gamma_{k}(f)\right)(x)-f(x)\right| \leq \omega_{1}\left(f, \frac{a+1}{2^{k}}\right) .
\end{gathered}
$$

Proof. (i) For $B_{k}$ operators: Here $\left(t_{o}(f)\right)(u)=f(u)$ and

$$
\sup _{\substack{u, y \in \mathbb{R} \\|u-y| \leq a}}\left|\left(t_{0}(f)\right)(u)-f(y)\right|=\sup _{\substack{u, y \in \mathbb{R} \\|u-y| \leq a}}|f(u)-f(y)|=w_{1}(f, a),
$$

and we use Theorem 3.4

(ii) For $\Gamma_{k}$ operators: Here we see that

$$
\sup _{\substack{u, y \in \mathbb{R} \\|u-y| \leq a}}\left|\left(t_{0}(f)\right)(u)-f(y)\right|=\sup _{\substack{u, y \in \mathbb{R} \\|u-y| \leq a}}\left|\sum_{j=0}^{n} w_{j} f\left(u+\frac{j}{n}\right)-f(y)\right| \leq
$$




$$
\begin{aligned}
& \sup _{\substack{u, y \in \mathbb{R} \\
|u-y| \leq a}} \sum_{j=0}^{n} w_{j}\left|f\left(u+\frac{j}{n}\right)-f(y)\right| \leq \sup _{\substack{u, y \in \mathbb{R} \\
|u-y| \leq a}} \sum_{j=0}^{n} w_{j} w_{1}\left(f,\left|u+\frac{j}{n}-y\right|\right) \leq \\
& \sup _{\substack{u, y \in \mathbb{R} \\
|u-y| \leq a}} \sum_{j=0}^{n} w_{j} \omega_{1}\left(f, \frac{j}{n}+|u-y|\right) \leq\left(\sum_{j=0}^{n} w_{j}\right) \omega_{1}(f, 1+\alpha)=\omega_{1}(f, \alpha+1) .
\end{aligned}
$$

By (29) we are done.

\section{Higher order of Approximation}

Here all are as in Section 3. See also earlier our work [1], and 2, Chapter 16.

We give

Theorem 4.1. Let $\mathrm{f} \in \mathrm{C}^{\mathrm{N}}\left(\mathbb{R}, \mathbb{R}_{+}\right), \mathrm{N} \geq 1$. Consider the Shilkret-sublinear operators

$$
\left(B_{k} f\right)(x)=\left(N^{*}\right) \int_{-a}^{a} f\left(x-\frac{u}{2^{k}}\right) \psi(u) d \mu(u)
$$

$\forall \mathrm{k} \in \mathbb{Z}, \forall x \in \mathbb{R}$. Then

$$
\left|\left(B_{k} f\right)(x)-f(x)\right| \leq \sum_{i=1}^{N} \frac{\left|f^{(i)}(x)\right|}{i !} \frac{a^{i}}{2^{k i}}+\frac{a^{N}}{2^{k N N} N !} \omega_{1}\left(f^{(N)}, \frac{a}{2^{k}}\right)
$$

If $\mathrm{f}^{(\mathrm{N})}$ is uniformly continuous or bounded and continuous, then as $\mathrm{k} \rightarrow+\infty$ we obtain that $\left(\mathrm{B}_{\mathrm{k}} \mathrm{f}\right)(\mathrm{x}) \rightarrow \mathrm{f}(\mathrm{x})$ pointwise with rates.

Proof. Since $f \in \mathrm{C}^{\mathrm{N}}\left(\mathbb{R}, \mathbb{R}_{+}\right), \mathrm{N} \geq 1$, by Taylor's formula we have

$$
\begin{gathered}
f\left(x-\frac{u}{2^{k}}\right)-f(x)=\sum_{i=1}^{N} \frac{f^{(i)}(x)}{i !}\left(-\frac{u}{2^{k}}\right)^{i}+ \\
\int_{x}^{x-\frac{u}{2^{k}}}\left(f^{(N)}(t)-f^{(N)}(x)\right) \frac{\left(x-\frac{u}{2^{k}}-t\right)^{N-1}}{(N-1) !} d t .
\end{gathered}
$$

Call

$$
\Gamma_{\mathrm{u}}(x):=\left|\int_{x}^{x-\frac{u}{2^{k}}}\left(f^{(N)}(t)-f^{(N)}(x)\right) \frac{\left(x-\frac{u}{2^{k}}-t\right)^{N-1}}{(N-1) !} d t\right| .
$$

Next we estimate $\Gamma_{u}(x)$, where $u \in[-a, a]$.

i) Case of $-a \leq u \leq 0$, then $x \leq x-\frac{u}{2^{k}}$. Then

$$
\Gamma_{u}(x) \leq \int_{x}^{x-\frac{u}{2^{k}}}\left|f^{(N)}(t)-f^{(N)}(x)\right| \frac{\left(x-\frac{u}{2^{k}}-t\right)^{N-1}}{(N-1) !} d t \leq
$$




$$
\begin{gathered}
\int_{x}^{x-\frac{u}{2^{k}}} \omega_{1}\left(f^{(N)},|t-x|\right) \frac{\left(x-\frac{u}{2^{k}}-t\right)^{N-1}}{(N-1) !} d t \leq \\
\omega_{1}\left(f^{(N)}, \frac{|u|}{2^{k}}\right) \int_{x}^{x-\frac{u}{2^{k}}} \frac{\left(x-\frac{u}{2^{k}}-t\right)^{N-1}}{(N-1) !} d t \leq \\
\omega_{1}\left(f^{(N)}, \frac{a}{2^{k}}\right) \frac{\left(-\frac{u}{2^{k}}\right)^{N}}{N !} \leq \omega_{1}\left(f^{(N)}, \frac{a}{2^{k}}\right) \frac{a^{N}}{2^{k N} N !} .
\end{gathered}
$$

That is, when $-a \leq u \leq 0$, then

$$
\Gamma_{\mathrm{u}}(\mathrm{x}) \leq \omega_{1}\left(f^{(\mathrm{N})}, \frac{\mathrm{a}}{2^{\mathrm{k}}}\right) \frac{\mathrm{a}^{\mathrm{N}}}{2^{\mathrm{kN} N !}} .
$$

ii) Case of $0 \leq u \leq a$, then $x \geq x-\frac{\mathfrak{u}}{2^{k}}$. Then

$$
\begin{gathered}
\Gamma_{u}(x)=\left|\int_{x-\frac{u}{2^{k}}}^{x}\left(f^{(N)}(t)-f^{(N)}(x)\right) \frac{\left(t-x+\frac{u}{2^{k}}\right)^{N-1}}{(N-1) !} d t\right| \leq \\
\int_{x-\frac{u}{2^{k}}}^{x}\left|f^{(N)}(t)-f^{(N)}(x)\right| \frac{\left(t-x+\frac{u}{2^{k}}\right)^{N-1}}{(N-1) !} d t \leq \\
\int_{x-\frac{u}{2^{k}}}^{x} \omega_{1}\left(f^{(N)},|t-x|\right) \frac{\left(t-x+\frac{u}{2^{k}}\right)^{N-1}}{(N-1) !} d t \leq \\
\omega_{1}\left(f^{(N)}, \frac{|u|}{2^{k}}\right) \int_{x-\frac{u}{2^{k}}}^{x} \frac{\left(t-x+\frac{u}{2^{k}}\right)^{N-1}}{(N-1) !} d t \leq \\
\omega_{1}\left(f^{(N)}, \frac{a}{2^{k}}\right) \frac{\left(\frac{u}{2^{k}}\right)^{N}}{N !} \leq \omega_{1}\left(f^{(N)}, \frac{a}{2^{k}}\right) \frac{a^{N}}{2^{k N} N !} .
\end{gathered}
$$

That is, when $0 \leq u \leq a$, then

$$
\Gamma_{u}(x) \leq \omega_{1}\left(f^{(N)}, \frac{a}{2^{k}}\right) \frac{a^{N}}{2^{k N N} N} .
$$

We proved that

$$
\Gamma_{\mathfrak{u}}(\mathrm{x}) \leq \omega_{1}\left(f^{(\mathrm{N})}, \frac{a}{2^{\mathrm{k}}}\right) \frac{a^{\mathrm{N}}}{2^{\mathrm{kN} N} !}:=\rho \geq 0,
$$

$\forall \mathrm{k} \in \mathbb{Z}, \forall x \in \mathbb{R},|\mathrm{u}| \leq \mathrm{a}$.

By (48) we get that $(|u| \leq a)$

$$
\left|f\left(x-\frac{u}{2^{k}}\right)-f(x)\right| \leq \sum_{i=1}^{N} \frac{\left|f^{(i)}(x)\right|}{i !} \frac{a^{i}}{2^{k i}}+\rho .
$$

We observe that

$$
\left|\left(B_{k} f\right)(x)-f(x)\right|=
$$




$$
\begin{gathered}
\left|\left(N^{*}\right) \int_{-a}^{a} f\left(x-\frac{u}{2^{k}}\right) \psi(u) d \mu(u)-\left(N^{*}\right) \int_{-a}^{a} f(x) \psi(u) d \mu(u)\right| \\
\left(N^{*}\right) \int_{-a}^{a}\left|f\left(x-\frac{u}{2^{k}}\right)-f(x)\right| \psi(u) d \mu(u) \leq \\
\left(\sum_{i=1}^{N} \frac{\left|f^{(i)}(x)\right|}{i !} \frac{a^{i}}{2^{k i}}+\rho\right)\left(\left(N^{*}\right) \int_{-a}^{a} \psi(u) d \mu(u)\right) \stackrel{(15)}{=} \\
\left(\sum_{i=1}^{N} \frac{\left|f^{(i)}(x)\right|}{i !} \frac{a^{i}}{2^{k i}}+\rho\right) \cdot 1= \\
\sum_{i=1}^{N} \frac{\left|f^{(i)}(x)\right|}{i !} \frac{a^{i}}{2^{k i}}+\frac{a^{N}}{2^{k N} N !} \omega_{1}\left(f^{(N)}, \frac{a}{2^{k}}\right)
\end{gathered}
$$

proving the claim.

Corollary 4.2. Let $\mathrm{f} \in \mathrm{C}^{1}\left(\mathbb{R}, \mathbb{R}_{+}\right)$. Then

$$
\left|\left(B_{k} f\right)(x)-f(x)\right| \leq \frac{a}{2^{k}}\left(\left|f^{\prime}(x)\right|+\omega_{1}\left(f^{\prime}, \frac{a}{2^{k}}\right)\right),
$$

$\forall \mathrm{k} \in \mathbb{Z}, \forall x \in \mathbb{R}$.

Proof. By (47) for $\mathrm{N}=1$.

We also present

Theorem 4.3. Let $\mathrm{f} \in \mathrm{C}^{\mathrm{N}}\left(\mathbb{R}, \mathbb{R}_{+}\right), \mathrm{N} \geq 1$. Consider the Shilkret-sublinear operators

$$
\left(\Gamma_{k}(f)\right)(x)=\left(N^{*}\right) \int_{-a}^{a}\left[\sum_{j=0}^{n} w_{j} f\left(\left(x-\frac{u}{2^{k}}\right)+\frac{j}{2^{k} n}\right)\right] \psi(u) d \mu(u),
$$

$\forall \mathrm{k} \in \mathbb{Z}, \forall x \in \mathbb{R}$. Then

$$
\left|\left(\Gamma_{k} f\right)(x)-f(x)\right| \leq \sum_{i=1}^{N} \frac{\left|f^{(i)}(x)\right|}{i !} \frac{(a+1)^{i}}{2^{k i}}+\frac{(a+1)^{N}}{N ! 2^{k N}} \omega_{1}\left(f^{(N)}, \frac{a+1}{2^{k}}\right) .
$$

If $\mathrm{f}^{(\mathrm{N})}$ is uniformly continuous or bounded and continuous, then as $\mathrm{k} \rightarrow+\infty$ we obtain that $\left(\Gamma_{\mathrm{k}} \mathrm{f}\right)(\mathrm{x}) \rightarrow \mathrm{f}(\mathrm{x})$, pointwise with rates.

Corollary 4.4. Let $\mathrm{f} \in \mathrm{C}^{1}\left(\mathbb{R}, \mathbb{R}_{+}\right)$. Then

$$
\left|\left(\Gamma_{k} f\right)(x)-f(x)\right| \leq \frac{(a+1)}{2^{k}}\left[\left|f^{\prime}(x)\right|+\omega_{1}\left(f^{\prime}, \frac{a+1}{2^{k}}\right)\right],
$$

$\forall \mathrm{k} \in \mathbb{Z}, \forall x \in \mathbb{R}$.

Proof. By (61) for $\mathrm{N}=1$. 
Proof. of Theorem 4.3 .

Since $f \in C^{N}(\mathbb{R}), N \geq 1$, by Taylor's formula we get

$$
\begin{gathered}
\sum_{j=0}^{n} w_{j} f\left(\left(x-\frac{u}{2^{k}}\right)+\frac{j}{2^{k} n}\right)-f(x)= \\
\sum_{i=1}^{N} \frac{f^{(i)}(x)}{i !} \sum_{j=0}^{n} w_{j}\left(-\frac{u}{2^{k}}+\frac{j}{2^{k} n}\right)^{i}+ \\
\sum_{j=0}^{n} w_{j} \int_{x}^{\left(x-\frac{u}{2^{k}}\right)+\frac{j}{2^{k} n}}\left(f^{(N)}(t)-f^{(N)}(x)\right) \frac{\left(\left(x-\frac{u}{2^{k}}\right)+\frac{j}{2^{k} n}-t\right)^{N-1}}{(N-1) !} d t .
\end{gathered}
$$

Call

$$
\varepsilon(x, u, j):=\int_{x}^{\left(x-\frac{u}{2^{k}}\right)+\frac{j}{2^{k} n}}\left(f^{(N)}(t)-f^{(N)}(x)\right) \frac{\left(\left(x-\frac{u}{2^{k}}\right)+\frac{j}{2^{k} n}-t\right)^{N-1}}{(N-1) !} d t .
$$

We estimate $\varepsilon(x, \mathfrak{u}, \mathfrak{j})$. Here $|\mathfrak{u}| \leq \mathrm{a}$.

i) case of $u \leq \frac{j}{n}$, iff $\frac{u}{2^{k}} \leq \frac{j}{2^{k} n}$, iff $x \leq x-\frac{u}{2^{k}}+\frac{j}{2^{k} n}$.

Hence

$$
\begin{gathered}
|\varepsilon(x, u, j)| \leq \int_{x}^{\left(x-\frac{u}{2^{k}}\right)+\frac{j}{2^{k} n}}\left|f^{(N)}(t)-f^{(N)}(x)\right| \frac{\left(\left(x-\frac{u}{2^{k}}\right)+\frac{j}{2^{k} n}-t\right)^{N-1}}{(N-1) !} d t \leq \\
\quad \int_{x}^{\left(x-\frac{u}{2^{k}}\right)+\frac{j}{2^{k_{n}}}} \omega_{1}\left(f^{(N)},|t-x|\right) \frac{\left(\left(x-\frac{u}{2^{k}}\right)+\frac{j}{2^{k} n}-t\right)^{N-1}}{(N-1) !} d t \leq \\
\omega_{1}\left(f^{(N)},\left[\frac{j}{2^{k} n}-\frac{u}{2^{k}}\right]\right) \int_{x}^{\left(x-\frac{u}{2^{k}}\right)+\frac{j}{2^{k} n}} \frac{\left(\left(x-\frac{u}{2^{k}}\right)+\frac{j}{2^{k} n}-t\right)^{N-1}}{(N-1) !} d t \leq \\
\omega_{1}\left(f^{(N)}, \frac{a+1}{2^{k}}\right) \frac{\left(\frac{j}{2^{k} n}-\frac{u}{2^{k}}\right)^{N}}{N !} \leq \omega_{1}\left(f^{(N)}, \frac{a+1}{2^{k}}\right) \frac{(a+1)^{N}}{2^{k N} N !} .
\end{gathered}
$$

For $u \leq \frac{j}{n}$, we hve proved that

$$
|\varepsilon(x, u, j)| \leq \omega_{1}\left(f^{(N)}, \frac{a+1}{2^{k}}\right) \frac{(a+1)^{N}}{2^{k N N} N !} .
$$

ii) case of $u \geq \frac{j}{n}$, iff $\frac{u}{2^{k}} \geq \frac{j}{2^{k} n}$, iff $x \geq x-\frac{u}{2^{k}}+\frac{j}{2^{k} n}$.

We observe that

$$
|\varepsilon(x, u, j)|=
$$




$$
\begin{aligned}
& \left|\int_{\left(x-\frac{u}{2^{k}}\right)+\frac{j}{2^{k_{n}}}}^{x}\left(f^{(N)}(t)-f^{(N)}(x)\right) \frac{\left(t-\left[\left(x-\frac{u}{2^{k}}\right)+\frac{j}{2^{k} n}\right]\right)^{N-1}}{(N-1) !} d t\right| \leq \\
& \int_{\left(x-\frac{u}{2^{k}}\right)+\frac{j}{2^{k_{n}}}}^{x}\left|f^{(N)}(t)-f^{(N)}(x)\right| \frac{\left(t-\left[\left(x-\frac{u}{2^{k}}\right)+\frac{j}{2^{k} n}\right]\right)^{N-1}}{(N-1) !} d t \leq \\
& \int_{\left(x-\frac{u}{2^{k}}\right)+\frac{j}{2^{k} n}}^{x} \omega_{1}\left(f^{(N)},|t-x|\right) \frac{\left(t-\left[\left(x-\frac{u}{2^{k}}\right)+\frac{j}{2^{k} n}\right]\right)^{N-1}}{(N-1) !} d t \leq \\
& \omega_{1}\left(f^{(N)}, \frac{u}{2^{k}}-\frac{j}{2^{k} n}\right) \int_{\left(x-\frac{u}{2^{k}}\right)+\frac{j}{2^{k} n}}^{x} \frac{\left(t-\left[\left(x-\frac{u}{2^{k}}\right)+\frac{j}{2^{k} n}\right]\right)^{N-1}}{(N-1) !} d t \leq \\
& \omega_{1}\left(f^{(N)}, \frac{a+1}{2^{k}}\right) \frac{\left(\frac{u}{2^{k}}-\frac{j}{2^{k} n}\right)^{N}}{N !} \leq \omega_{1}\left(f^{(N)}, \frac{a+1}{2^{k}}\right) \frac{(a+1)^{N}}{2^{k N N} N} .
\end{aligned}
$$

So when $u \geq \frac{j}{n}$, we proved that

$$
|\varepsilon(x, u, j)| \leq \omega_{1}\left(f^{(N)}, \frac{a+1}{2^{k}}\right) \frac{(a+1)^{N}}{2^{k N N} N !} .
$$

Therefore it always holds

$$
|\varepsilon(x, u, j)| \leq \omega_{1}\left(f^{(N)}, \frac{a+1}{2^{k}}\right) \frac{(a+1)^{N}}{2^{k N N} .} .
$$

Consequently we derive

$$
\sum_{j=0}^{n} w_{j}|\varepsilon(x, u, j)| \leq w_{1}\left(f^{(N)}, \frac{a+1}{2^{k}}\right) \frac{(a+1)^{N}}{2^{k N} N !}:=\bar{\psi} .
$$

By (63) we find

$$
\left|\sum_{j=0}^{n} w_{j} f\left(\left(x-\frac{u}{2^{k}}\right)+\frac{j}{2^{k} n}\right)-f(x)\right| \leq \sum_{i=1}^{N} \frac{\left|f^{(i)}(x)\right|}{i !} \frac{(a+1)^{i}}{2^{k i}}+\bar{\psi} .
$$

Therefore we get

$$
\begin{gathered}
\left|\left(\Gamma_{k}(f)\right)(x)-f(x)\right|= \\
\left|\left(N^{*}\right) \int_{-a}^{a}\left[\sum_{j=0}^{n} w_{j} f\left(\left(x-\frac{u}{2^{k}}\right)+\frac{j}{2^{k} n}\right)\right] \psi(u) d \mu(u)-\left(N^{*}\right) \int_{-a}^{a} f(x) \psi(u) d \mu(u)\right| \stackrel{(20)}{\leq} \\
\left(N^{*}\right) \int_{-a}^{a}\left|\sum_{j=0}^{n} w_{j} f\left(\left(x-\frac{u}{2^{k}}\right)+\frac{j}{2^{k} n}\right)-f(x)\right| \psi(u) d \mu(u) \stackrel{(73)}{\leq}
\end{gathered}
$$




$$
\begin{gathered}
{\left[\sum_{i=1}^{N} \frac{\left|f^{(i)}(x)\right|}{i !} \frac{(a+1)^{i}}{2^{k i}}+\bar{\psi}\right]\left(N^{*}\right) \int_{-a}^{a} \psi(u) d \mu(u) \stackrel{(15)}{=}} \\
{\left[\sum_{i=1}^{N} \frac{\left|f^{(i)}(x)\right|}{i !} \frac{(a+1)^{i}}{2^{k i}}+\bar{\psi}\right] \cdot 1=} \\
\sum_{i=1}^{N} \frac{\left|f^{(i)}(x)\right|}{i !} \frac{(a+1)^{i}}{2^{k i}}+\frac{(a+1)^{N}}{2^{k N} N !} \omega_{1}\left(f^{(N)}, \frac{a+1}{2^{k}}\right),
\end{gathered}
$$

proving the claim.

We finish with

Corollary 4.5. Let $f \in \mathrm{C}^{\mathrm{N}}\left(\mathbb{R}, \mathbb{R}_{+}\right), \mathrm{N} \geq 1, \mathrm{f}^{(i)}(\mathrm{x})=0, i=1, \ldots, \mathrm{N}$. Then

i)

$$
\left|\left(B_{k}(f)\right)(x)-f(x)\right| \leq \frac{a^{N}}{2^{k N} N !} \omega_{1}\left(f^{(N)}, \frac{a}{2^{k}}\right),
$$

and

ii)

$$
\left|\left(\Gamma_{k}(f)\right)(x)-f(x)\right| \leq \frac{(a+1)^{N}}{N ! 2^{k N}} \omega_{1}\left(f^{(N)}, \frac{a+1}{2^{k}}\right),
$$

$\forall \mathrm{k} \in \mathbb{Z}, \forall x \in \mathbb{R}$.

Proof. By (47) and (61).

Corollary 4.6. Let $f \in \mathrm{C}^{1}\left(\mathbb{R}, \mathbb{R}_{+}\right), \mathrm{f}^{\prime}(\mathrm{x})=0$. Then

i)

$$
\left|\left(B_{k}(f)\right)(x)-f(x)\right| \leq \frac{a}{2^{k}} \omega_{1}\left(f^{\prime}, \frac{a}{2^{k}}\right),
$$

and

ii)

$$
\left|\left(\Gamma_{k}(f)\right)(x)-f(x)\right| \leq\left(\frac{a+1}{2^{k}}\right) \omega_{1}\left(f^{\prime}, \frac{a+1}{2^{k}}\right),
$$

$\forall \mathrm{k} \in \mathbb{Z}, \forall x \in \mathbb{R}$.

Proof. By (59) and (62).

In inequalities (76)-(79) observe the high speed of convergence and approximation. 


\section{Appendix}

Let $f \in C_{u}\left(\mathbb{R}, \mathbb{R}_{+}\right)$, and the positive sublinear Shilkret operator

$$
(M(f))(x):=\left(N^{*}\right) \int_{-a}^{a} f(x+u) \psi(u) d \mu(u), \quad \forall x \in \mathbb{R} .
$$

We observe the following (for any $x, y \in \mathbb{R}$ ):

$$
\begin{gathered}
|(M(f))(x)-(M(f))(y)|= \\
\left|\left(N^{*}\right) \int_{-a}^{a} f(x+u) \psi(u) d \mu(u)-\left(N^{*}\right) \int_{-a}^{a} f(y+u) \psi(u) d \mu(u)\right| \stackrel{\sqrt[200]{\leq}}{\left(N^{*}\right) \int_{-a}^{a}|f(x+u)-f(y+u)| \psi(u) d \mu(u) \leq} \\
\omega_{1}(f,|x-y|)\left(\left(N^{*}\right) \int_{-a}^{a} \psi(u) d \mu(u)\right) \stackrel{(15)}{=} \omega_{1}(f,|x-y|) \cdot 1=\omega_{1}(f,|x-y|) .
\end{gathered}
$$

Therefore it holds the global smoothness preservation property:

$$
\omega_{1}(M(f), \delta) \leq \omega_{1}(f, \delta), \forall \delta>0 .
$$

\section{References}

[1] G.A. Anastassiou, High order Approximation by univariate shift-invariant integral operators, in: R. Agarwal, D. O’Regan (eds.), Nonlinear Analysis and Applications, 2 volumes, vol. I, pp. 141-164, Kluwer, Dordrecht, (2003).

[2] G.A. Anastassiou, Intelligent Mathematics: Computational Analysis, Springer, Heidelberg, New York, 2011.

[3] G.A. Anastassiou, S. Gal, Approximation Theory, Birkhauser, Boston, Basel, Berlin, 2000.

[4] G.A. Anastassiou, H.H. Gonska, On some shift invariant integral operators, univariate case, Ann. Polon. Math., LXI, 3, (1995), 225-243.

[5] Niel Shilkret, Maxitive measure and integration, Indagationes Mathematicae, 33 (1971), 109116. 